\title{
Structure diversity of transition metal coordination compounds based on pyridine derivative co-ligands
}

\author{
Merrill Margaret Wicht \\ Cape Peninsula University of Technology, Cape Town, South Africa; \\ wichtm@cput.ac.za
}

The synthesis of transition metal complexes with central metals $\mathrm{Ni}(\mathrm{II}), \mathrm{Co}(\mathrm{II})$ and $\mathrm{Zn}$ (II) with thiocyanate or chloride anions and pyridine derivative co-ligands according to the formula of Werner complexes $\mathrm{MX}_{2} \mathrm{~L}_{4}$ presented structural diversity. The 'tunability' of the crystal structures arises from the transformation of the nature and size of the inclusion cavities. In general, octahedral coordination complexes occur with nickel and cobalt. However, zinc showed a preference for tetrahedral coordination, resulting in $\mathrm{MX}_{2} \mathrm{~L}_{2}$ crystals, where $\mathrm{M}$ is the central metal, $\mathrm{X}$ the anion and $\mathrm{L}$ the pyridine derivative ligand.

The effect of the position of derivatives on the pyridine ring (meta- or para-) altered the interaction between the host molecules forming a variety of frameworks. In the case of nickel and cobalt, the nicotinamide ligand (meta position amide) a linear arrangement of the ligands occurs resulting in a predictable and robust framework which shows hydrogen bonding of amide tetramers. Steric hindrance between the derivatives results in torsioning of the ligands if the derivative is para to the pyridine nitrogen. Hosts $\mathrm{Ni}(\mathrm{NCS})_{2}$ and $\mathrm{Co}(\mathrm{NCS})_{2}$ with four isonicotinamide ligands presented a variety of frameworks ranging from spiral format to herringbone arrangement. The trans ligands present a propeller arrangement which disables the predictability of the framework.

A further discovery was made with mixed ligand complexes. The prominence of amide dimer formation via hydrogen bonds between nicotinamide ligands was emphasised yet isonicotinamide ligands showed only discrete hydrogen bonds. Sulphur hydrogen bonds O$\mathrm{H} \cdots \mathrm{S}$ in nickel clathrates resulted in better thermal stability. This case was observed with $\mathrm{Ni}(\mathrm{NCS})_{2}$ (nicotinamide) $)_{4}$ clathrates with an alcohol guest compared with those with carbonyl guest.

Discrimination between two guests was shown by nickel complexes for a number of equimolar guests, notably the selection of 1butanol from an equimolar mixture with 2-pentanol; 4-methylcyclohexanone from a mixture of 3- and 4-methylcyclohexanone; and propanol was selected over isopropanol. The host $\mathrm{Ni}(\mathrm{NCS})_{2}$ (4-phenylpyridine $)_{2}$ (isoquinoline) $)_{2}$ targets meta-xylene over ortho- and para-xylene in an equimolar mixture of the isomers. Stronger $\mathrm{C}-\mathrm{H} \cdots \pi$ intermolecular interactions were found in the Hirshfeld surface analysis between the host and meta-xylene than in the other two isomers.

The versatility of the central metal in these Werner complexes was investigated by measuring the thermal properties of the release of the guest from isotypic clathrates using differential scanning and thermogravimetric analysis. The diversity of the complexes and the versatility of the ligands in these Werner complexes demonstrates their importance in the discriminatory ability of guest mixtures.

Keywords: crystal structures, coordination compounds, thermal properties, hydrogen bonding 